The weight of the extracted bullet (126 grains) accurately corresponded with the weight of one of the bullets left in the revolver.
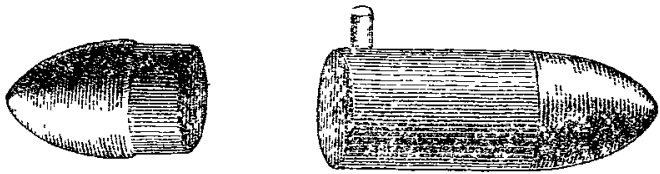

These figures show one of the bullets with, the other without, its metallic cartridge; all the illustrations being of natural size.

The extraction was effected without violence, once the elerator was placed under the foreign body and worked with my left hand, while the right index-finger acted as a fulcrum. Nothing was introduced into the ear, and little blood was lost. Free vent was afterwards allowed for discharge, which was collected in absorbent antiseptic pads, specially prepared by Messrs. Southall Brothers and Barclay. As the discharge was fetid, the pads acted agreeably as deodorisers, and their downy softness was so comfortable, that the patient's repeated wish was for more pads. At first ice was applied to the head by an ordinary ice-bag; but, as the temperature rose, a continuous current of iced water cooled the head through one of Galante's syphon tube caps, which, fortunately, Mr. Salt had just previously brought over from Paris. The effect of this appliance was very speedily to reduce the temperature two degrees; but though there was unremitting care under the immediate supervision of $m y$ house-surgeon, Mr. Westwood Moore, cerebral compression ended in death forty-two hours after the operation.

Birmingham.

\section{A CASE OF STRANGULATED CONGENITAL HERNIA IN A CHILD FOURTEEN MONTHS} OLD ; OPERATION ; RECOVERY.

\section{BY IVILLIAM MONTAGUE BALL, M.B.}

THE chief points of interest in this case consist in the early age of the patient and in the closure, after the operation, of the internal abdominal ring to a sufficient extent to prevent re-descent of the intestine.

On Feb. 22nd, at 8 P.M., the child, a fine little boy, fourteen months old, was brought to my house, very pale, and evidently in pain. The mother stated he had been ruptured since birth, but that, having been always able to "put it back," she had neglected the advice of procuring a truss; that morning, however, the bowel had come down during a fit of crying, and she had not been able to return it. She also said he had been sick several times.

Upon examination I found a swelling extending along the right inguinal canal into the scrotum, tense, tender, and with no impulse on crying. Having tried taxis for a reasonable time, both in and out of a warm bath, I left the child for the nicht, after giving it a small dose of opium, slung up in a kind of hammock, with its hips much elevated and an ice-bag on the tumour.

The next morning, at eight o'clock, still being unable to effect reduction, I asked my friend Dr. Sydney to administer chloroform, and finding the hernia still irreducible, proceded to operate in the usual way, using Lister's steamspray and his antiseptic precautions. On reaching the sac it was found distended with a small quantity of dark-coloured Huid; the intestine was a good deal congested and showed evidence of strangulation. The seat of stricture was at the internal abdominal ring. Owing to the child being very fat considerable difficulty was experienced in dividing the stricture to a sulficient extent to allow of the bowel being returned ; eventually this was accomplished and the wound brought together with catgut sutures, and dressed antiseptically. The child was ordered to be fed on milk and lime-water, and half a drachm of tincture of catechu was administered. In spite of astringents the bowels acted copiously within twentyfrour hours, and the child vomited once during the night. The dressings were removed in forty-eight hours, and the wound was found to be quite healthy and closed at the lower end. Temperature $100 \cdot 2^{\circ} \mathrm{F}$.

Feb. 26th.-Patient passed a very restless night, moaning and crying a great deal. Wound again dressed, the deeper portion connected with the abdominal cavity quite closed, the more superficial part, however, had gaped somewhat, owing doubtless to the difficulty of keeping so young a child from wriggling about, notwithstanding it was confined in a box-splint. The abdomen was very tympanitic and very tender. Temperature $103^{\circ}$; pulse 180. Bran poultices to be constantly applied and one grain of compound ipecacuanha powder with a quarter of a grain of calomel to be given every four hours. This treatment was carried on for some days, the opium keeping the child free from pain ; and although but little hope was entertained of recovery, eventually the tympanites disappeared and tenderness over the abdomen ceased.

The wound on the fifth day was found to be suppurating freely, and so was dressed with carbolic oil in gauze. A sinus extending four inches in the direction of the right hypochondrium formed and had to be pressed out twice daily. After the first movement of the bowels, they acted once or twice daily during the progress of the case. The child was able to take the breast throughout its illness.

March 28th. - Wound quite healed and patient allowed to crawl about with a pad-and-spica bandage.

April 3rd. - A truss was applied and the child able to run about once more.

July 10th. - I saw the child running about, and the mother tells me it has been quite well ever since, and that there has been no appearance of any return of the rupture, although the truss has occasionally been left off for some time. I presume that during the attack of peritonitis which succeeded the operation, enough coagulable lymph was thrown out to procure a sufficient closure of the internal abdominal ring to prevent any re-descent of the bowel.

Hounslow.

\section{GUNSHOT WOUND OF THE MEDIAN NERVE.}

BY EDWARD LAWRIE, M.B.,

PROFESSOR OF SURGERY, LAHORE MEDICAL SCHOOL.

IN the early part of May this year a boy named G. Caged ten, was accidentally struck in the right arm by a charge of small shot. Several pellets were removed by his medical attendant from underneath the skin in the neighbourhood of the elbow, but none appeared to have penetrated more deeply. A good deal of superficial inflammation followed, and the boy lost the use of his forearm and hand. It is not clear from the account of the friends whether this paralysis was immediate or came on gradually. He suffered great and increasing pain in the arm and corresponding side of the neck and body, and was brought to me July 27 th, 1880 . I found a very peculiar drop of the right wrist and loss of power of the forearm, together with exalted sensibility in the palm of the hand. There was much glandular and lymphatic enlargement, which obscured the diagnosis, but thickening of the median nerve was satisfactorily made out about the middle of the arm, and I therefore cut down upon it. When exposed, the nerve was more than twice its proper size, and I was about to stretch it when I detected a hard body in its interior. Instead of stretching, a longitudinal incision was made into its substance and a pellet found and removed. As the operation was performed antiseptically, the wound healed by first intention; the boy rapidly recovered power in the forearm and normal sensation in the hand, and was discharged cured on August 6th, 1880

Lahore.

The Parkes Museum of Hygiene.-This museum was reopened last Monday (after the usual vacation) in connexion with the introductory conversazione of the Medical School at University College. There was a good attendance of gentlemen and students, who manifested much interest in the collection of sanitary appliances, to which several additions have recently been made, including concrete and stoneware goods from the Poole pottery district. Messrs. Tonks and Son have added to their contribution a model of a new ventilator, called "the architrave ventilator," designed by Mr. Mark H. Judge, for the purpose of making an architectural feature of the provisions for ventilation. The Parkes Museum will continue to be open free on Tuesdays, Thursdays, and Saturdajs. 